\title{
DEPOLARIZATION CHANNEL FOR BARCELONA LIDAR. IMPLEMENTATION AND PRELIMINARY MEASUREMENTS.
}

\author{
Alejandro Rodríguez-Gómez ${ }^{1 *}$, Michaël Sicard ${ }^{1,2}$, Constantino Muñoz-Porcar ${ }^{1}$, Rubén Barragán $^{1,2}$, \\ Adolfo Comerón $^{1}$, Francesc Rocadenbosch ${ }^{1,2}$, Eric Vidal ${ }^{3}$ \\ ${ }^{1}$ RSLab, BarcelonaTech University (UPC), Barcelona, Spain*alejandro@tsc.upc.edu \\ ${ }^{2}$ Ciències i Tecnologies de l'Espai - Centre de Recerca de l'Aeronàutica i de l'Espai / Institut d'Estudis \\ Espacials de Catalunya (CTE-CRAE / IEEC), UPC, Barcelona, Spain \\ ${ }^{3}$ UTC Building \& Industrial Systems, Spain
}

\begin{abstract}
A new depolarization channel has been implemented in the BarcelonaTech University (UPC) multi-wavelength lidar system. The optical and mechanical designs are presented. The special configuration of the total power channel is also detailed, with the relevant aspects in measurement inversion. Some preliminary measurements are presented for Saharan dust intrusion events.
\end{abstract}

\section{INTRODUCTION}

The capability of measuring depolarization of the backscattered light, together with multiwavelength detection, improves the ability of a lidar system to identify the aerosols present in the atmosphere, as it has been pointed out by different authors [1]-[4].

The BarcelonaTech University (UPC) Remote Sensing Lab (RSLab) has implemented a new depolarization channel for its lidar system. The depolarization channel has been tested with measurements during Saharan dust intrusion events taking place on May and June 2016.

\section{THE UPC LIDAR MAIN RECEIVER CONFIGURATION}

The lidar system at UPC [5] presents a noncommon receiver configuration that is sketched in Figure 1. A 14-inch telescope collects the backscattered light, which is delivered to the polychromator unit by means of a 3-m long multimode fiber bundle of $3 \mathrm{~mm}$ diameter. The polychromator delivers light to the 6 photo-receivers tuned at 1064, 532 and $355 \mathrm{~nm}$ (elastic backscattering) and 607, 407 and $387 \mathrm{~nm}$ (Raman channels).
According to fiber optics theory [6], a multi-mode fiber will not preserve the polarization of the guided light.

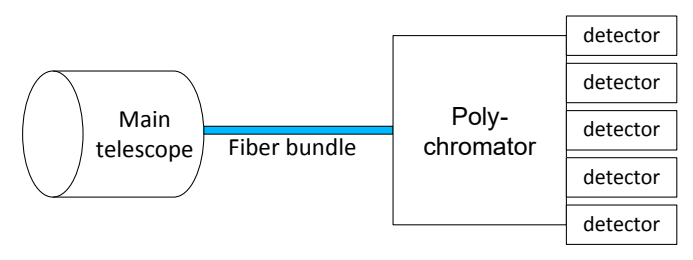

Figure 1 UPC lidar receiver configuration, including a depolarizing multi-mode fiber bundle.

This fact makes that the overall behavior of the receiver is non-sensitive to the polarization of the incoming light. So the main receiver becomes total-power collecting.

To check this feature, we measured the depolarization introduced by the fiber bundle, with a simple test set, presented in Figure 2. The laser beam passes through a linear polarizer before being fed to the fiber bundle.

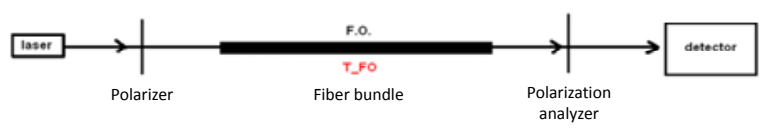

Figure 2 Test set for checking the polarization behavior of the fiber bundle

The polarization state of the output light was measured in two different situations: with the fiber bundle cable placed straight and rolled up. The results are plotted in Figure 3. The measured circularities are 0.96 for the cable straight and 0.93 for the cable rolled up.

This depolarization capability is also useful to make our system insensitive to the di-attenuation of the different beam-splitters used in the polychromator unit. 


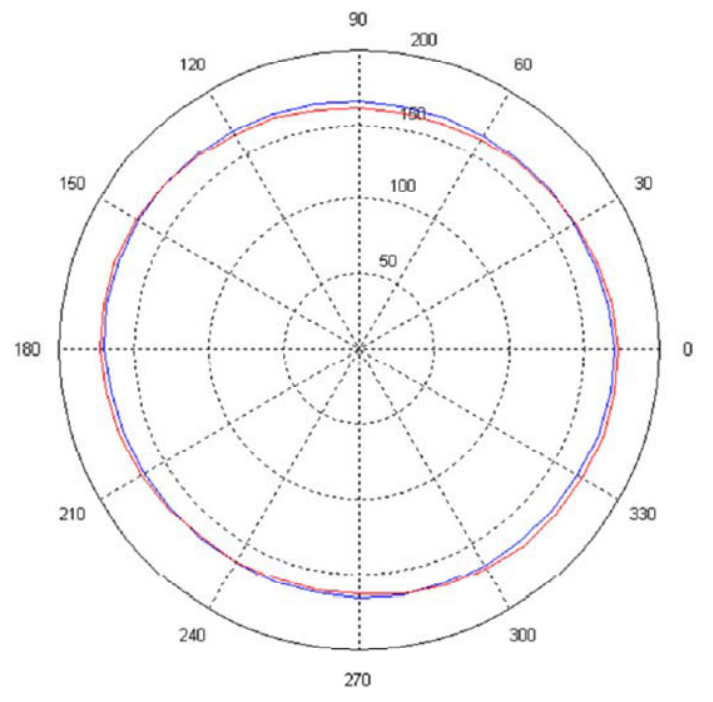

Figure 3 Polarization state measurement at the output of the fiber bundle cable for two situations: straight (blue line) and rolled up (red line) cable.[7]

\section{NEW DEPOLARIZATION CHANNEL}

The depolarization channel has been built by means of an auxiliary telescope attached to the lidar mechanical setup. The auxiliary telescope is, in fact, a 300-mm focal distance, $70-\mathrm{mm}$ aperture diameter, TAIR-3S telephoto lens.

The optical setup, sketched in Figure 4, includes an eye-piece lens that produces an image of the telescope aperture onto the photo-receiver (a Licel R9880U PMT module) sensitive area. This arrangement allows distributing the collected light over the PMT sensitive surface, while a $1-\mathrm{mm}$ field-stop iris limits the field of view to approx. $3.3 \mathrm{mrad}$.

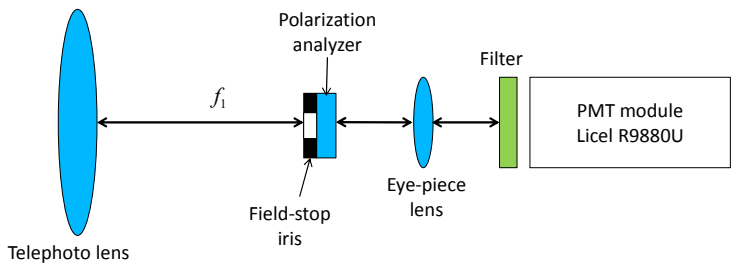

Figure 4 Optical setup of the depolarization channel

A polarization analyzer located at the focal plane of the telephoto lens selects the desired polarization of the collected light. When performing measurements, the position of the analyzer is oriented to collect the cross-polar component; when performing the needed calibration of the channel [4] the analyzer is oriented alternatively at +45 and -45 degrees from the nominal position.

Finally, an interference filter at $532 \mathrm{~nm}$ is located near the photo-receiver surface. According to this arrangement, the collected light rays are not parallel when passing through the filter. This fact implies that the filter effective spectral width is greater than the specified value $(0.5 \mathrm{~nm})$. This has implications especially in the molecular contribution to the depolarized backscattered light [8] and must be taken into account when processing the measurement results.

\section{PRINCIPLE OF OPERATION [4]}

The primary depolarization measurement is done by comparing the signals detected by the depolarization and total power channels

$$
\begin{aligned}
& S_{D e p}\left(90^{\circ}, R\right)=V_{D e p}(R) \cdot P_{\perp}(R) \\
& S_{\text {Tot }}(R)=V_{\text {Tot }}(R) \cdot P_{\text {Tot }}(R)
\end{aligned}
$$

where $P_{\perp}(R)$ and $P_{\text {Tot }}(R)$ are the cross-polar and total powers collected while $V_{\text {Dep }}(R)$ and $V_{\text {Tot }}(R)$ are the depolarization and total power channels responsivities, as a function of height over the lidar $R$. The primary observable is

$$
\delta^{*}\left(90^{\circ}, R\right)=\frac{S_{D e p}\left(90^{\circ}, R\right)}{S_{T O T}(R)}
$$

In parallel to the measurement process, a channel calibration (with respect to the total power channel) must be performed to obtain the system channel ratio

$$
V^{*}(R)=\frac{V_{D e p}(R)}{V_{T o t}(R)}
$$

The calibration is done by performing two short measurements with the polarization analyzer rotated +45 and -45 degrees from the nominal position, $\quad S_{D e p}\left(90^{\circ}+45^{\circ}, R\right) \quad$ and $S_{D e p}\left(90^{\circ}-45^{\circ}, R\right)$. The system calibration profile is then computed by comparing with the signal obtained simultaneously from the total power channel, $S_{T o t}(R)$ : 


$$
V^{*}(R)=2 \sqrt{\frac{S_{D e p 1}\left(90^{\circ}-45^{\circ}, R\right)}{S_{T o t 1}(R)} \cdot \frac{S_{D e p 2}\left(90^{\circ}+45^{\circ}, R\right)}{S_{T o t 2}(R)}}
$$

Figure 5 shows the result of a calibration performed on December $2^{\text {nd }} 2016$. As it can be noted, a spatial average is performed

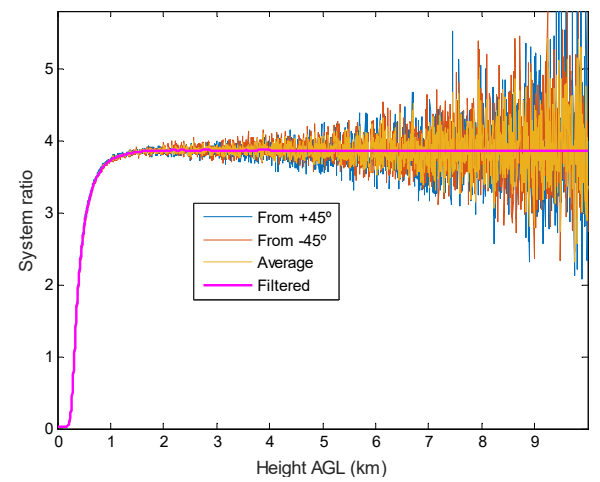

Figure 5 System channel-ratio calibration performed on December $2^{\text {nd }} 2016$.

The volume depolarization ratio is then computed

$$
\delta^{V}(R)=\frac{\delta^{*}\left(90^{\circ}, R\right)}{V^{*}(R)-\delta^{*}\left(90^{\circ}, R\right)}
$$

Finally, the particle depolarization ratio is computed by using concurrently the results of the molecular $\beta^{m}(R)$ and particle $\beta^{p}(R)$ backscattering coefficients of the atmosphere under measure, via an elastic or Raman inversion of the total power 532-nm channel:

$$
\delta^{p}(R)=\frac{\left[1+\delta^{m}(R)\right] \cdot \delta^{V}(R) \cdot \rho(R)-\left[1+\delta^{V}(R)\right] \delta^{m}(R)}{\left[1+\delta^{m}(R)\right] \cdot \rho(R)-\left[1+\delta^{V}(R)\right]}
$$

where:

$$
\rho(R)=\frac{\beta^{m}(R)+\beta^{p}(R)}{\beta^{m}(R)}
$$

and $\delta^{m}(R)$, the molecular volume depolarization ratio is usually considered a constant, which depends on the temperature and the spectral width of the photo-receiver [8].

\section{RESULTS}

Two Saharan dust intrusion events took place around Barcelona area in late May and early June 2016. Measurements were performed with the UPC lidar and simultaneously with a co-located Micro Pulse Lidar, associated to MPLnet [9]. This Micro Pulse Lidar provides the range-corrected total power and depolarization signals and calculates the volume depolarization in real time.

Figure 6 and Figure 7 show two consecutive measurements during May $26^{\text {th }}$. They show a Saharan dust layer around $3.7 \mathrm{~km}$ altitude, with particle depolarization ratio exceeding $20 \%$ (more than $15 \%$ is characteristic of this kind of aerosol). Both measurements show good agreement between the UPC lidar and the MPL.

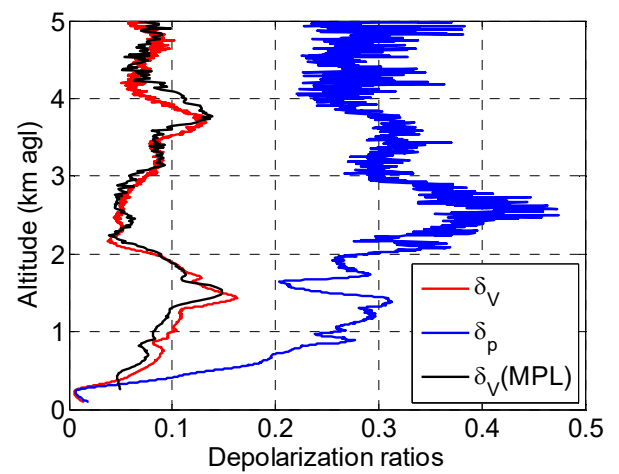

Figure 6 Measurement performed on May $26^{\text {th }} 2016$, 14:24 UTC

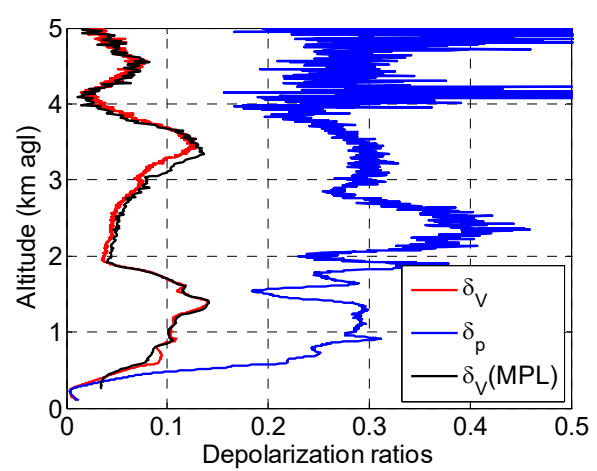

Figure 7 Measurement performed on May 26 2016 , 15:40 UTC.

Figure 8 and Figure 9 show measurements taken, respectively, in the beginning and in the maximum of an intrusion event in June 2016. Figure 8 shows a small value of volume depolarization up to $5 \mathrm{~km}$, while Figure 9 shows a great mass of aerosol between 3 and $4 \mathrm{~km}$ height, 
also with particle depolarization over $20 \%$. In this latest measurement, there is a noticeable difference with the MPL measurement, very likely due to some mechanical tolerance in the telescope pointing not considered because of the absence of a calibration on the day of the measurement.

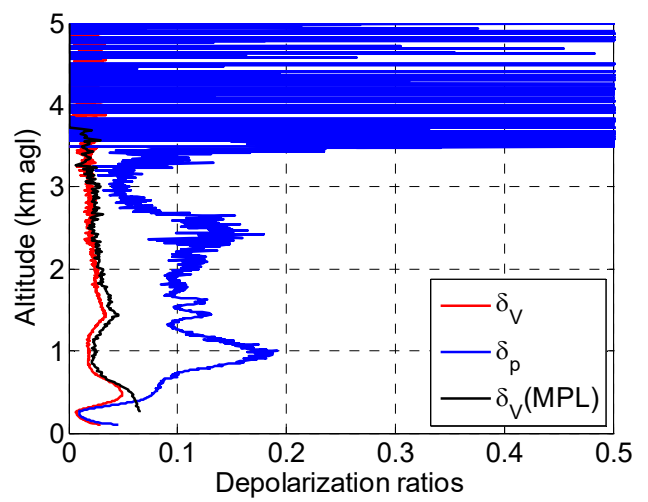

Figure 8 Measurement performed on June $7^{\text {th }} 2016$, 8:37 UTC.

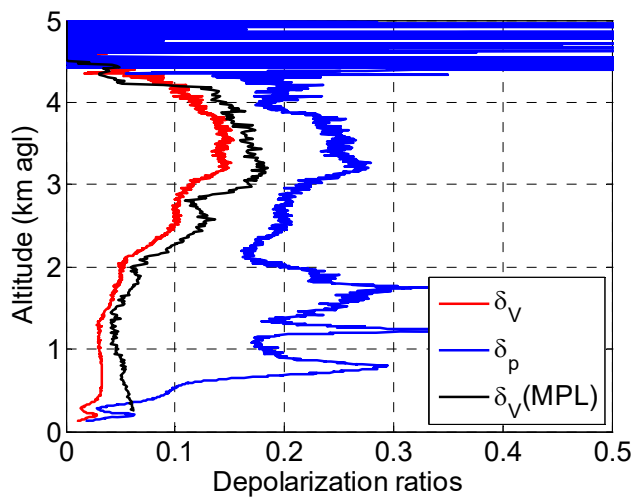

Figure 9 Measurement performed on June $10^{\text {th }} 2016$, 13:17 UTC

\section{CONCLUSIONS}

The new depolarization channel of the UPC lidar has been introduced with details of architecture and principle of operation. Measurements performed during two Saharan dust intrusion events are used to test the new channel. Particle depolarization ratios varying between 10 and 40 $\%$ are found in the dust layers. Comparisons of the volume depolarization with an MPL system show a good agreement between both systems and demonstrate the reliability of the new depolarization channel of the UPC multiwavelength lidar.

\section{ACKNOWLEDGEMENTS}

This work has been financed by the European Union (H2020, grant 654169, ACTRIS-2), the European Fund for Regional Development, the Spanish Government (grant TEC2015-63832-P) and the Catalan Government (grant 2014 SGR 583).

The authors also acknowledge the priceless help of Joaquim Giner and Josep Pastor, both members of UPC technical staff.

\section{References}

[1] K. Sassen, "Polarization in Lidar," in Lidar, C. Weitkamp, Ed. New York: Springer-Verlag, 2005, pp. $19-42$.

[2] K. Sassen, J. Zhu, P. Webley, K. Dean, and P. Cobb, "Volcanic ash plume identification using polarization lidar: Augustine eruption, Alaska," Geophys. Res. Lett., vol. 34, no. 8, Apr. 2007.

[3] G. Wandinger, Ulla; Ansmann, Albert; Mattis, Ina; Müller, Detlef; Pappalardo, "Calipso and beyond: long-term ground-based support of space-borne aerosols and cloud lidar missions" in 24th International Laser Radar Conference, 2008, pp. 715-718.

[4] V. Freudenthaler et al., "Depolarization ratio profiling at several wavelengths in pure Saharan dust during SAMUM 2006," Tellus, Ser. B Chem. Phys. Meteorol., vol. 61, no. 1, pp. 165-179, 2009.

[5] D. Kumar et al., "Six-channel polychromator design and implementation for the UPC elastic/Raman lidar," SPIE Int. Symp. - Remote Sens. Eur., vol. 8182, p. 81820W-1-81820W-10, 2011.

[6] G. P. Agrawal, Fiber-Optic Communications Systems, Third Edition., vol. 6. 2002.

[7] E. Vidal, "Disseny d'un canal de despolarització a $532 \mathrm{~nm}$ per al lidar d'EARLINET de la UPC," BarcelonaTech, 2013.

[8] A. Behrendt and T. Nakamura, "Calculation of the calibration constant of polarization lidar and its dependency on atmospheric temperature," Opt. Express, vol. 10, no. 16, p. 805, Aug. 2002.

[9] NASA, “MPLNet," 2017. [Online]. Available: https://mplnet.gsfc.nasa.gov/. [Accessed: 11-Jan2017]. 